\title{
Relação entre as causas de morte evitáveis por atenção à saúde e a implementação do Sistema Único de Saúde no Brasil
}

\author{
Daisy Maria Xavier de Abreu, ${ }^{1}$ Cibele Comini César ${ }^{2}$ \\ e Elisabeth Barboza França ${ }^{3}$
}

Como citar Abreu DMX, César CC, França EB. Relação entre as causas de morte evitáveis por atenção à saúde e a implementação do Sistema Único de Saúde no Brasil. Rev Panam Salud Publica. 2007;21(5):282-91.

RESUMO Objetivos. Analisar a relação entre a ocorrência de mortes que poderiam ser evitadas por atenção à saúde e o processo de reorganização do sistema de saúde brasileiro entre 1983 e 2002. Métodos. No presente estudo ecológico, a mortalidade por causas evitáveis foi analisada em 117 municípios. As causas de morte evitáveis por atenção à saúde foram agrupadas em: evitáveis por diagnóstico e tratamento precoce, evitáveis por melhoria no tratamento e na atenção médica e doença isquêmica do coração. Para avaliar a associação entre as causas de morte evitáveis e a reorganização do sistema de saúde, o período analisado foi dividido em dois subperíodos, 1983 a 1992 e 1993 a 2002 (antes e depois da aprovação da norma operacional que serviu como referencial para a implantação do Sistema Único de Saúde). Utilizou-se um modelo de regressão binomial negativa, com controle das variáveis sexo, idade, região geográfica e condições socioeconômicas.

Resultados. No período analisado, ocorreram 1854165 óbitos por causas evitáveis nas idades de 0 a 74 anos nos municípios selecionados. A análise multivariada indicou que o risco foi maior no período de 1983 a 1992 em relação ao período de 1993 a 2002 para os três grupos de causas evitáveis estudados. Observou-se que os homens apresentaram risco maior, particularmente para a doença isquêmica do coração. As populações mais jovens tiveram um risco menor. O nível socioeconômico mais elevado reduziu o risco de morte por causas evitáveis, exceto para a doença isquêmica do coração.

Conclusões. Os resultados sugerem que, no Brasil, o declínio da mortalidade por causas evitáveis entre 1983 e 2002 deveu-se, em parte, às mudanças na oferta e no acesso aos serviços de saúde, impulsionadas pela reorganização do sistema de saúde a partir da década de 1990.

Palavras-chave Avaliação de serviços de saúde, Brasil, causas de morte evitáveis, estudos ecológicos, mortalidade.

Universidade Federal de Minas Gerais (UFMG), Faculdade de Medicina, Programa de Pós-Graduação em Saúde Pública. Enviar correspondência para esta autora no seguinte endereço: Av. Alfredo Balena 190, sala 9028, Santa Efigênia, CEP 30130-100, Belo Horizonte, MG, Brasil. Tel: +55-31-3248-9685; e-mail: dmxa@medicina.ufmg.br

2 Universidade Federal de Minas Gerais (UFMG), Instituto de Ciências Exatas, Departamento de Estatística, Belo Horizonte (MG), Brasil.
O interesse em avaliar o impacto dos sistemas de atenção à saúde sobre os níveis de saúde da população foi impulsionado por Rutstein et al. (1), que

\footnotetext{
3 Universidade Federal de Minas Gerais (UFMG), Faculdade de Medicina, Departamento de Medicina Preventiva e Social, Belo Horizonte (MG), Brasil.
}

identificaram grupos de causas de óbito relacionados à qualidade da atenção prestada. Essa abordagem considera que, se o progresso tecnológico e médico-científico e o modelo assistencial são direcionados para a prevenção e o tratamento de doenças evitáveis, o desenvolvimento desse tipo de doença 
poderia não ocorrer ou ser interrompido em estágios menos avançados. Dessa forma, seria possível reduzir as taxas de morbidade hospitalar e de mortalidade por essas causas.

Ao final do século $X X$ e início do século XXI, a validade e a aplicabilidade do conceito de mortalidade evitável como uma medida de efeito da atenção à saúde foram demonstradas em vários estudos conduzidos em países desenvolvidos (2-7). Desde então, verificou-se um crescimento do interesse científico na identificação, mensuração e análise de variações temporais, regionais e socioeconômicas da mortalidade por patologias que poderiam ser evitadas por ações de saúde. Nesses estudos, observa-se uma variabilidade nas metodologias aplicadas, nos critérios de seleção de causas de morte e nas variáveis explicativas e de confusão utilizadas (8). A definição de quais causas de morte podem ser consideradas como evitáveis tem se modificado no decorrer do tempo em função do desenvolvimento e da introdução de novas tecnologias médicas, bem como dos objetivos dos estudos realizados (9).

Os trabalhos que abordam tendências temporais sugerem que a redução nos níveis de mortalidade por causas evitáveis pode ser explicada, pelo menos em parte, pelo aumento da efetividade dos serviços de saúde (10-12). O ritmo de declínio pode também estar relacionado com a introdução de uma melhoria específica da atenção médica (13). Outros estudos comparativos têm indicado que variações regionais existentes na mortalidade podem estar relacionadas ao acesso à assistência, que reflete as condições socioeconômicas da população $(2,3,14$, 15). As melhorias na oferta e no acesso a serviços de saúde de qualidade tiveram impacto em vários países durante os anos 1980 e 1990, com redução da mortalidade de crianças, de adultos em algumas faixas etárias e de mulheres em idades mais avançadas (8).

No Brasil, a análise de causas evitáveis para a população em geral ainda é pouco desenvolvida $(16,17)$. O critério de evitabilidade da mortalidade, baseado na classificação de Rutstein et al. (1), vem sendo explorado, na maioria das vezes, em estudos sobre mortalidade infantil e perinatal (18-20). Entretanto, a abordagem da mortalidade evitável na realidade brasileira se torna pertinente em virtude dos padrões de morbi-mortalidade vigentes, que exigem respostas das políticas públicas de saúde nos diferentes níveis de atenção. Ainda persistem significativas diferenças entre regiões e municípios brasileiros, tanto na distribuição da oferta de atenção à saúde quanto no acesso da população aos serviços de saúde. Essas diferenças se refletem na saúde da população e repercutem nos níveis e na estrutura da mortalidade, mantendo, ampliando ou reduzindo os diferenciais regionais e sociais $(21,22)$. Portanto, as políticas de saúde devem ser capazes de enfrentar esse desafio.

$\mathrm{O}$ atual sistema de saúde brasileiro apresenta, como um de seus principais objetivos, alterar a situação de desigualdade na oferta de serviços de saúde à população. Sua organização foi engendrada a partir de um longo processo cumulativo de transformações políticas mais amplas, incrementadas na década de 1980 (23). Várias propostas de organização da atenção à saúde antecederam e orientaram o modelo consubstanciado na Constituição Federal de 1988, que criou o Sistema Único de Saúde (SUS). Esse sistema buscou organizar uma rede assistencial que possibilitasse o acesso universal e igualitário às ações e serviços de saúde, com um modelo baseado na regionalização e hierarquização do cuidado, na priorização de atividades preventivas e no desenvolvimento de mecanismos de participação da comunidade (24). O processo de mudança teve início efetivo nos anos 1990, com atos normativos e administrativos que procuraram garantir a acessibilidade da população aos serviços de saúde, com repercus-são nos indicadores de saúde da população (25).

Nesse contexto, justifica-se o desenvolvimento de estudos para identificar as implicações das mudanças ocorridas a partir da implementação do SUS para a saúde da população brasileira. Ao se ampliar o acesso aos serviços de saúde, seria esperada uma redução nas causas de morte evitáveis por atenção à saúde. Dessa forma, o presente trabalho teve como objetivo analisar a mortalidade por causas evitáveis em municípios brasileiros no período de 1983 a 2002, relacionando-a com o processo de reorganização do sistema de saúde brasileiro.

\section{MATERIAIS E MÉTODOS}

Este é um estudo ecológico, cuja unidade de análise é o município. Foram investigados os óbitos por causas evitáveis no período de 1983 a 2002. A fonte de dados para óbitos foi o Sistema de Informações sobre Mortalidade (SIM) do Ministério da Saúde. As estimativas populacionais foram obtidas junto ao Instituto Brasileiro de Geografia e Estatística (IBGE). Os dados foram processados pelo programa Tabwin 3.2, com programação dos arquivos de conversão e definição para município, sexo, idade e causas selecionadas (baseadas na Nona Revisão da Classificação Internacional de Doenças, CID-9, para os anos de 1983 a 1995, e na Décima Revisão, CID-10, para o período de 1996 a 2002). Considerando-se que o uso de duas versões da CID poderia representar um problema para a análise da evolução da mortalidade por causas $(8,26)$, a definição das causas evitáveis baseou-se nos códigos de três dígitos, o que garantiu que as mesmas patologias fossem incluídas na classificação de causas evitáveis utilizada.

\section{Definição da amostra}

Em virtude dos problemas de qualidade das informações de óbitos ainda existentes no Brasil (27-29), foram definidos os seguintes critérios de inclusão para seleção dos municípios deste estudo: população maior do que $100 \mathrm{mil}$ habitantes, população urbana maior do que $90 \%$, desvio médio da taxa geral de mortalidade (DTGM) menor do que $10 \%$, porcentagem de causas mal definidas menor do que $10 \%$.

A escolha desses critérios deveu-se ao fato de que municípios maiores e mais urbanizados dispõem de melho- 
res condições em termos da oferta de serviços de saúde e melhor qualidade nas informações. Quanto ao DTGM, valores menores do que $10 \%$ indicam maior estabilidade das taxas, com controle de possíveis flutuações estocásticas. Além desses, um dos indicadores clássicos da qualidade das informações sobre mortalidade é a proporção de óbitos atribuídos a causas mal definidas, que deve ser menor do que $10 \%$ (26).

A partir desses critérios, foram incluídos 117 municípios brasileiros. A grande maioria encontra-se nas regiões Sudeste e Sul (84 e 24 municípios, respectivamente, o que representa cerca de $92 \%$ dos 117 municípios), com poucos municípios localizados no Nor- deste e Centro-Oeste (cinco e quatro municípios, respectivamente). $\mathrm{Ne}$ nhum município da Região Norte foi selecionado.

\section{Variáveis selecionadas}

Para definir as causas evitáveis de óbito, considerou-se a revisão das classificações de causas evitáveis realizada por Nolte e McKee (8) e as propostas de Simonato et al. (30) e Tobias e Jackson (31). Esta última ampliou e qualificou as classificações desenvolvidas por Charlton et al. (2) e Mackenbach et al. (12). Foi construída uma tipologia adaptada de causas evitáveis, com três grupamentos (tabela 1).
O primeiro grupo foi constituído pelas causas evitáveis por diagnóstico e tratamento precoce, incluindo causas de morte para as quais há evidências de que cuidados médicos preventivos podem postergar ou impedir o avanço da doença (neoplasmas de mama, útero, pele, dentre outras) ou a recorrência dos eventos (acidente vascular cerebral, por exemplo). O segundo grupo foi composto por causas consideradas evitáveis por melhoria no tratamento e na atenção médica. Neste grupamento foram incluídas, por exemplo, as infecções intestinais, cuja mortalidade é altamente afetada por tratamento com antibióticos e vacinação. Também fazem parte deste grupo, em virtude da sua evitabilidade

TABELA 1. Classificação das causas evitáveis de morte em grupos ${ }^{a}$

\begin{tabular}{|c|c|c|c|}
\hline Grupo de causas & Idade (anos) & CID $9^{b}$ & $\operatorname{CID} 10^{c}$ \\
\hline \multicolumn{4}{|l|}{ Grupo de causas evitáveis por diagnóstico e tratamento precoce } \\
\hline Neoplasma maligno do cólon e reto & 0 a 74 & $153-154$ & $\mathrm{C} 18-\mathrm{C} 21$ \\
\hline Neoplasma maligno da pele & 0 a 74 & 173 & C44 \\
\hline Neoplasma maligno da mama & 0 a 74 & 174 & C50 \\
\hline Neoplasma maligno do colo do útero & 0 a 74 & 180 & C53 \\
\hline Neoplasma maligno do corpo do útero & 0 a 44 & 179,182 & C54, C55 \\
\hline Doenças da tireóide & 0 a 74 & $240-246$ & E00-E07 \\
\hline Diabetes melito & 0 a 74 & 250 & E10-E14 \\
\hline Epilepsia & 0 a 74 & 345 & G40-G41 \\
\hline Doenças cerebrovasculares & 0 a 74 & $430-438$ & $160-169$ \\
\hline Influenza & 0 a 74 & 487 & J10-J11 \\
\hline Pneumonia & 0 a 74 & $480-486$ & J12-J18 \\
\hline Hiperplasia da próstata & 0 a 74 & 600 & N40 \\
\hline \multicolumn{4}{|l|}{ Grupo de causas evitáveis por melhoria no tratamento e na atenção médica } \\
\hline Infecções intestinais & 0 a 14 & 001-009 & A00-A09 \\
\hline Tuberculose & 0 a 74 & $010-018,137$ & A15-A19, B90 \\
\hline Outras infecções (difteria, tétano, poliomielite) & 0 a 74 & $032,037,045$ & $\mathrm{~A} 36, \mathrm{~A} 35, \mathrm{~A} 80$ \\
\hline Coqueluche & 0 a 14 & 033 & A37 \\
\hline Septicemia & 0 a 74 & 38 & A40-A41 \\
\hline Sarampo & 1 a 14 & 55 & B05 \\
\hline Neoplasma maligno do testículo & 0 a 74 & 186 & C62 \\
\hline Doença de Hodgkin & 0 a 74 & 201 & C81 \\
\hline Leucemia & 0 a 74 & 204-208 & C91-C95 \\
\hline Doença reumática crônica do coração & 0 a 74 & 393-398 & $105-109$ \\
\hline Doenças hipertensivas & 0 a 74 & $401-405$ & $|10-| 13, \mid 15$ \\
\hline Todas as doenças respiratórias (exceto pneumonia/influenza) & 1 a 14 & $460-479,488-519$ & J00-J09, J20-J99 \\
\hline Úlcera péptica & 0 a 74 & $531-533$ & K25-K27 \\
\hline Apendicite & 0 a 74 & $540-543$ & K35-K38 \\
\hline Hérnia abdominal & 0 a 74 & $550-553$ & $\mathrm{~K} 40-\mathrm{K} 46$ \\
\hline Colelitíase e colecistite & 0 a 74 & $574-575.1$ & $\mathrm{~K} 80-\mathrm{K} 81$ \\
\hline Nefrite e nefrose & 0 a 74 & $580-589$ & N00-N07, N17-N19, N25-N27 \\
\hline Mortalidade materna & 0 a 74 & $630-676$ & 000-099 \\
\hline Anomalias congênitas do coração & 0 a 74 & $745-747$ & Q20-Q28 \\
\hline Acidentes com pacientes durante intervenção cirúrgica e atenção médica & 0 a 74 & E870-E876, E878-E879 & Y60-Y69, Y83-Y84 \\
\hline Doença isquêmica do coração & 0 a 74 & $410-414$ & $120-125$ \\
\hline
\end{tabular}

a Seleção baseada em classificações propostas por Simonato et al. (30), Tobias e Jackson (31) e Nolte e McKee (8).

b Nona revisão da Classificação Internacional de Doenças (1983 a 1992).

c Décima revisão da Classificação Internacional de Doenças (1993 a 2002). 
por intervenções médicas e cirúrgicas, as doenças hipertensivas, a úlcera péptica, a apendicite e a complicações da gravidez e do parto. A mortalidade por essas causas está relacionada com uma complexa articulação no interior do sistema de saúde, que prevê um diagnóstico acurado, acesso a serviços especializados e adequada atenção médica e cirúrgica (30). Assim, no segundo grupo, foram incluídas causas para as quais as taxas de mortalidade podem ser significativamente reduzidas por tratamento médico e cirúrgico existente, mesmo quando o processo da doença esteja totalmente desenvolvido. $\mathrm{O}$ terceiro grupo correspondeu à doença isquêmica do coração, considerada em separado devido ao seu peso relativo na mortalidade geral. Além disso, não há consenso sobre qual é o tipo de intervenção médica que prioritariamente tem impacto na ocorrência dessa doença (8).

A seleção das causas de morte evitáveis procurou abarcar diferentes tipos de atenção à saúde. $\mathrm{Na}$ escolha das causas foram excluídas aquelas para as quais a intervenção efetiva ocorre fora do controle direto do sistema de saúde, incluindo várias formas de prevenção primária, conforme proposto por Charlton et al. (2) e por Mackenbach et al. (12). Foram observados ainda os grupos etários para os quais cada patologia pode ser classificada como potencialmente evitável. A idade limite considerada para a maioria das causas foi 74 anos (tabela 1), tendo em vista as tendências de aumento da esperança de vida ao nascer e a limitação na possibilidade de intervenção visando a redução das taxas de mortalidade acima dessa faixa etária (8).

Neste estudo, não foram incluídas as causas perinatais, por falta de dados disponíveis sobre nascidos vivos para anos anteriores a 1994. Sabe-se que o enfoque da evitabilidade é recomendado para esses óbitos, dado que boa parte deles, especialmente em países em desenvolvimento, poderia ser evitada com a melhoria da assistência prénatal, ao parto e ao recém-nascido (20, 32). No Brasil, já existe uma discussão sobre o tema, no sentido de se elaborar uma classificação que possa ser utili- zada para esse grupo etário, em especial, e para as demais idades (33).

Foram selecionadas as seguintes variáveis socioeconômicas: percentual de pessoas com idade igual ou maior do que 25 anos e 12 anos ou mais de estudo; percentual de pessoas com idade igual ou maior do que 25 anos e menos de 4 anos de estudo; razão entre a renda média dos $10 \%$ mais ricos e a dos $40 \%$ mais pobres (medida do grau de desigualdade na distribuição de indivíduos segundo a renda domiciliar per capita); índice de Gini; renda per capita; e esperança de vida ao nascer. As variáveis socioeconômicas foram obtidas do Atlas do Desenvolvimento Humano no Brasil, com indicadores para os anos de 1991 e 2000, disponíveis por município. Esses anos correspondem aos dois últimos censos demográficos brasileiros (34).

Como foram utilizadas variáveis socioeconômicas possivelmente correlacionadas, e para evitar problemas de estimação do modelo, foi aplicada a técnica de análise fatorial para criação de novos indicadores socioeconômicos. A partir da análise dos interrelacionamentos das variáveis, foram gerados dois fatores, que explicavam $68 \%$ da variação observada nas variáveis originais. $\mathrm{O}$ primeiro fator apresentou correlação alta com as variáveis índice de Gini e razão entre a renda média dos $10 \%$ mais ricos e dos $40 \%$ mais pobres, e recebeu o nome de "desigualdade" (D). Já o segundo fator era altamente correlacionado com as variáveis percentual de pessoas com idade igual ou maior do que 25 anos e 12 anos ou mais de estudo, percentual de pessoas com idade igual ou maior do que 25 anos e menos de 4 anos de estudo e renda per capita, e foi denominado "nível socioeconômico" (NSE). A questão da raça/etnia não foi introduzida no modelo de análise por problemas de qualidade da informação disponível.

No controle de possíveis fatores de confusão, foram consideradas ainda as variáveis sexo, faixa etária (dividida em 0 a 14 anos, 15 a 44 anos, 45 a 64 anos e 65 a 74 anos) e região geográfica brasileira (Nordeste, Sudeste, CentroOeste e Sul).

\section{Análise dos dados}

Os dados foram analisados utilizandose o programa estatístico Stata 8.0.

$\mathrm{Na}$ análise da mortalidade por causas, foi considerado o número de óbitos por grupo de causas evitáveis no período de 1983 a 2002. Para o cálculo das taxas de mortalidade padronizadas por idade, foi utilizada, como distribuição etária padrão, a população do Brasil em 1991. Essa escolha baseou-se na consideração de que a população brasileira nesse ano poderia representar uma média razoável das distribuições etárias proporcionais existentes no país.

As análises de associação foram realizadas inicialmente através do modelo de regressão de Poisson. Na aplicação do modelo de Poisson, supõe-se igualdade entre a média e a variância. Entretanto, quando a variabilidade é maior do que a média, considera-se que há sobredispersão, ou seja, a suposição básica do modelo - de igualdade entre a média e a variância — não é válida (35). Uma alternativa nesse caso é o modelo binomial negativo, que considera a sobredispersão como resultado de uma heterogeneidade não observada. O modelo binomial negativo oferece como parâmetro a razão de taxas de incidência (incidence rate ratio, IRR), da mesma forma que o modelo de Poisson. O IRR indica qual foi a alteração na incidência (expressa em \%) associada ao acréscimo unitário na variável explicativa em análise, com todas as outras variáveis do modelo mantidas constantes.

Para todos os conjuntos de variáveis explicativas considerados, foram ajustados o modelo de Poisson e o modelo binomial negativo e, através do teste da razão de verossimilhança, verificouse a significância do parâmetro indicativo de heterogeneidade não observada $(\alpha)$. Em todos os casos, a hipótese de nulidade do parâmetro $\alpha$ foi rejeitada. Dessa forma, a análise final foi realizada através do modelo binomial negativo, que foi ajustado para identificar mudanças no nível e na variação da mortalidade evitável. A variável resposta foi o número de óbitos segundo as causas evitáveis seleciona- 
das. As variáveis explicativas selecionadas foram período (1983 a 1992 e 1993 a 2002), distribuição da população por sexo e faixa etária, região brasileira e condições socioeconômicas. $\mathrm{O}$ critério de significância adotado foi $P<0,05$.

A separação em dois períodos reflete a hipótese de que a mortalidade por causas evitáveis pode ter sido afetada pelas mudanças operadas no sistema de saúde em determinados momentos. Foi considerada como marcador dessas mudanças a introdução de normas que redefiniram a organização de serviços de saúde (25), ou seja, portarias do Ministério da Saúde que estabeleceram os objetivos e as diretrizes estratégicas para o processo de descentralização das políticas de saúde. A Norma Operacional SUS 01, de maio de 1993 (NOB-SUS 01/93), pode ser considerada como referencial do processo de implantação do SUS, na medida em que procurou sistematizar o processo de descentralização da gestão do sistema e serviços de saúde, transferindo para os estados e municípios as responsabilidades de operacionalização do SUS, antes concentradas no nível federal.

\section{RESULTADOS}

De 1983 a 2002, ocorreram 1854165 óbitos por causas evitáveis na faixa de 0 a 74 anos de idade nos municípios selecionados. Na população masculina, esses óbitos representaram 32,51\% do total de óbitos por todas as causas (1 016331 óbitos por causas evitáveis de 3126492 óbitos por todas as causas) e, na população feminina, $46,00 \%$ (837 834 óbitos por causas evitáveis de 1821526 óbitos por todas as causas) (tabela 2).

As causas evitáveis por diagnóstico e tratamento precoce responderam por $45,28 \%$ e $55,84 \%$ do total de óbitos por causas evitáveis para homens e mulheres, respectivamente. Dentre essas, as doenças cerebrovasculares foram as mais freqüentes, em ambos os sexos. As causas evitáveis por melhoria no tratamento e na atenção médica foram responsáveis por cerca de um quarto dos óbitos evitáveis em homens e mu- lheres. Nesse grupo de causas, as doenças hipertensivas foram as que tiveram a maior participação para ambos os sexos. Já a doença isquêmica do coração foi responsável por $29,68 \%$ dos óbitos masculinos e 19,66\% dos femininos (tabela 2).

Em termos da razão de taxas de mortalidade entre os dois períodos de análise, observou-se que o risco foi maior para o período de 1983 a 1992 em relação ao período de 1993 a 2002 para todos os grupos de causas evitáveis. O mesmo não ocorreu com as taxas de morte por causas não evitáveis, que se mantiveram praticamente constantes, tanto para os homens quanto para as mulheres (tabela 3).

A redução dos níveis de mortalidade pode também ser confirmada pela análise da variação percentual das taxas padronizadas por idade para os grupos de causas evitáveis nos dois períodos (tabela 3). Os resultados indicaram que, tanto para homens como para mulheres, houve redução dos níveis de mortalidade. A variação foi semelhante para as causas evitáveis por diagnóstico e tratamento e doença isquêmica do coração e mais evidente para as causas evitáveis por melhoria no tratamento e atenção médica. As maiores diferenças entre homens e mulheres em termos de níveis de mortalidade foram observadas para a doença isquêmica do coração, sendo que as taxas padronizadas para as mulheres representaram praticamente a metade daquelas encontradas para os homens, diferença mantida mesmo com o declínio das taxas de mortalidade por essa causa. No tocante à mortalidade por causas não evitáveis, as taxas padronizadas apresentaram um pequeno aumento para os homens (variação de 1,37\%), enquanto que para as mulheres houve uma diminuição de 5,31\%. No total das causas de morte, o que se observou foi uma redução nas taxas de mortalidade, sendo essa redução um pouco maior para as mulheres $(16,11 \%$ contra $10,48 \%$ para os homens). Nota-se ainda que as taxas padronizadas de mortalidade das mulheres representaram praticamente a metade das taxas dos homens para as causas não evitáveis e para o total de causas (tabela 3).
Na tabela 4 são apresentados os resultados das análises de associação entre a mortalidade para os grupos de causas evitáveis e variáveis explicativas selecionadas. Para cada grupo de causas evitáveis foi ajustado um modelo, incluindo todas as variáveis consideradas no estudo. Através desse modelo, foi possível identificar as variáveis significativas nos diferentes grupamentos de causas evitáveis.

A variável explicativa de maior interesse —período de análise (1983 a 1992 e 1993 a 2002) - manteve-se significativa no modelo final $(P<0,05)$ para os três grupos de causas evitáveis por atenção à saúde, após controle da situação socioeconômica, região geográfica, sexo e idade. $\mathrm{O}$ risco de morte por causas evitáveis por diagnóstico e tratamento precoce foi 33,56\% maior no período de 1983 a 1992 em relação ao período de 1993 a 2002. Para os óbitos por causas evitáveis por melhoria no tratamento e atenção médica, o risco foi maior cerca de 27,18\%. Para as mortes provocadas por doença isquêmica do coração, o risco foi maior 22,34\% no primeiro período (1983 a 1992) comparativamente ao segundo (1993 a 2002) (tabela 4).

A variável sexo apresentou uma performance de magnitude variada, mas sempre no mesmo sentido: o sexo masculino representou um risco maior em relação ao sexo feminino. Os homens tiveram um risco $14,03 \%$ maior para as mortes por causas evitáveis por diagnóstico e tratamento, 30,37\% maior para os óbitos por causas evitáveis por melhoria no tratamento e atenção médica e até $116,51 \%$ maior para doença isquêmica do coração.

A análise dos grupos etários em relação ao grupo de referência (65 a 74 anos) mostra um risco menor para as idades mais jovens (entre 0 e 14 anos e 15 e 44 anos). Esse comportamento foi ainda mais acentuado para os óbitos por doença isquêmica do coração.

A variável indicadora de localização regional (tomando a Região Sul como referência, por ser uma das regiões com melhores condições socioeconômicas), apresentou também situações particulares segundo o grupamento de causas evitáveis analisado. Essa variá- 
TABELA 2. Distribuição dos óbitos por causas evitáveis segundo o sexo, Brasil, 1983 a 2002

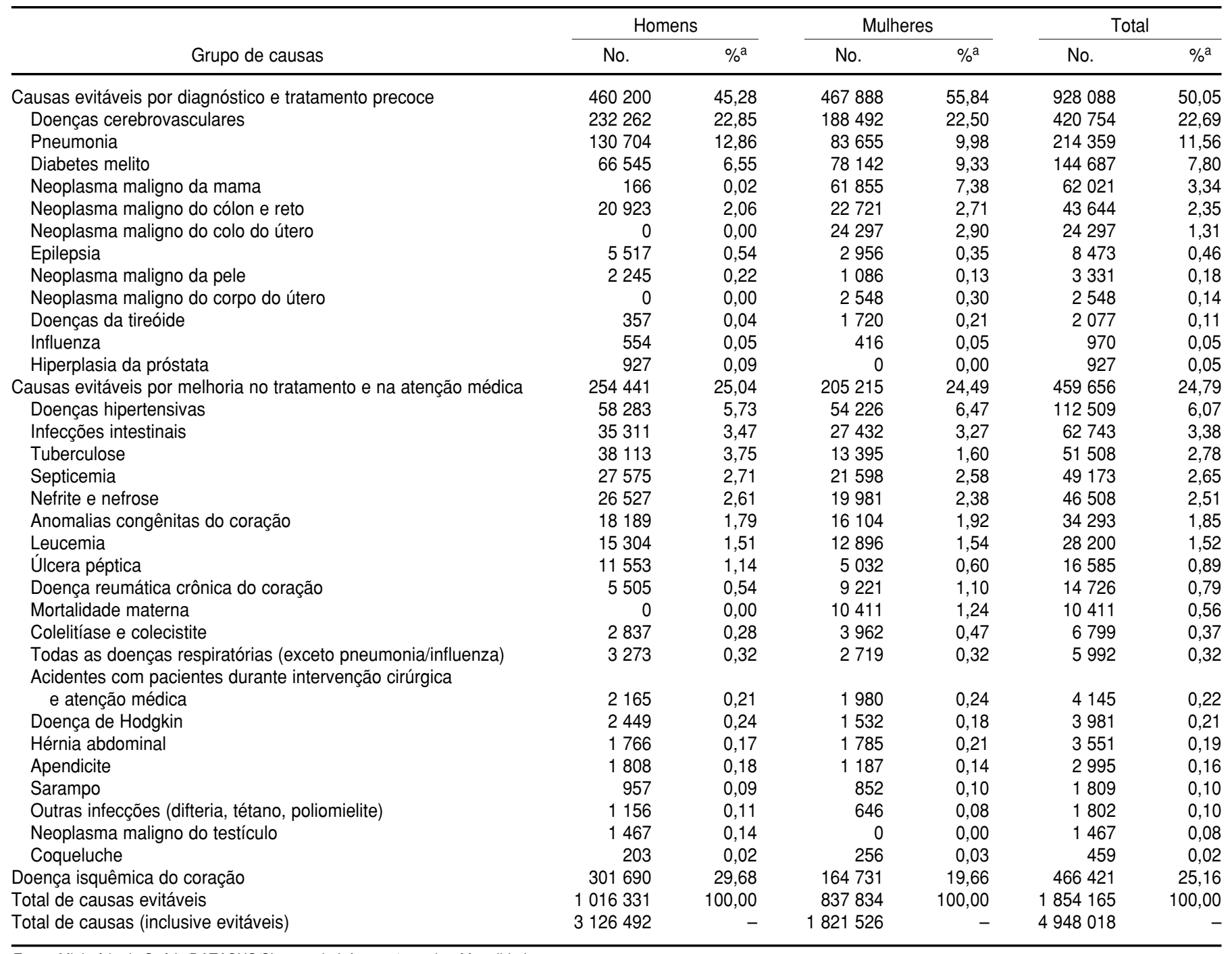

Fonte: Ministério da Saúde/DATASUS/Sistema de Informações sobre Mortalidade.

a Percentual em relação ao total de causas evitáveis por sexo.

vel só apresentou significância estatística (após controle do período, condição socioeconômica, sexo e idade) para os óbitos por doença isquêmica do coração, com todas as regiões apresentando risco menor em relação à Região Sul (tabela 4). Foi expressivo o valor do IRR encontrado para a Região Nordeste no caso das causas evitáveis por melhoria no tratamento e atenção médica - 47,53\% maior do que o risco de morte por esse grupo de causas na Região Sul. Já para os óbitos por causas evitáveis por diagnóstico e tratamento precoce, o risco foi $4,42 \%$ maior para o Sudeste quando comparado com a Região Sul.
Dentre os dois fatores indicadores de nível socioeconômico (NSE e D), o primeiro foi significativo para os óbitos por causas evitáveis por diagnóstico e tratamento e causas evitáveis por melhoria no tratamento e atenção médica. Dessa forma, sendo NSE uma variável contínua, pode-se inferir que, para cada aumento neste fator, houve uma redução no risco de morte por causas evitáveis de aproximadamente $8 \%$ para as mortes por causas evitáveis por diagnóstico e tratamento e de $10 \%$ para os óbitos por causas evitáveis por melhoria no tratamento e atenção médica. O fator $\mathrm{D}$, indicativo de desigualdade social, só não apresentou efeito significativo para os óbitos por causas evitáveis por diagnóstico e tratamento. Entretanto, os limites do intervalo de confiança de 95\% apresentaram valores muito próximos de 1 .

\section{DISCUSSÃO}

Os resultados deste estudo indicam que houve uma redução nos níveis de mortalidade por causas evitáveis por atenção à saúde no período de 1993 a 2002 em relação ao período de 1983 a 1992, após controle do nível socioeconômico e de variáveis demográficas. Para o grupamento de causas evitáveis 
TABELA 3. Distribuição das taxas de mortalidade padronizadas por idade segundo o grupo de causas evitáveis e o sexo, Brasil, 1983 a 1992 e 1993 a $2002^{\text {a }}$

\begin{tabular}{|c|c|c|c|c|c|c|c|c|}
\hline \multirow[b]{2}{*}{ Grupos de causas } & \multicolumn{4}{|c|}{ Homens } & \multicolumn{4}{|c|}{ Mulheres } \\
\hline & $\begin{array}{c}1983 \text { a } \\
1992 \\
\text { (a) }\end{array}$ & $\begin{array}{c}1993 a \\
2002 \\
\text { (b) }\end{array}$ & $\begin{array}{c}\text { Razão } \\
\text { de taxas } \\
(a / b)\end{array}$ & $\begin{array}{c}\text { Variação } \\
(\%)\end{array}$ & $\begin{array}{c}1983 \mathrm{a} \\
1992 \\
\text { (a) }\end{array}$ & $\begin{array}{c}1993 \mathrm{a} \\
2002 \\
\text { (b) }\end{array}$ & $\begin{array}{c}\text { Razão } \\
\text { de taxas } \\
\text { (a/b) }\end{array}$ & $\begin{array}{c}\text { Variação } \\
(\%)\end{array}$ \\
\hline \multicolumn{9}{|l|}{ Causas evitáveis por diagnóstico } \\
\hline Causas evitáveis por melhoria & & & & & & & & \\
\hline no tratamento e na atenção médica & 64,58 & 44,37 & 1,46 & $-31,29$ & 48,09 & 33,16 & 1,45 & $-31,05$ \\
\hline Total de causas & 677,19 & 606,25 & 1,12 & $-10,48$ & 375,76 & 315,23 & 1,19 & $-16,11$ \\
\hline
\end{tabular}

a Taxa de mortalidade por 100000 habitantes, idade entre 0 e 74 anos.

TABELA 4. Regressão binomial negativa para a mortalidade por grupos de causas evitáveis, Brasil

\begin{tabular}{|c|c|c|c|c|c|c|}
\hline \multirow[b]{2}{*}{ Variáveis } & \multicolumn{2}{|c|}{$\begin{array}{c}\text { Causas evitáveis por diagnóstico } \\
\text { e tratamento precoce }\end{array}$} & \multicolumn{2}{|c|}{$\begin{array}{l}\text { Causas evitáveis por melhoria } \\
\text { no tratamento e na atenção médica }\end{array}$} & \multicolumn{2}{|c|}{ Doença isquêmica do coração } \\
\hline & IRR $^{\mathrm{a}}$ & (IC95\%) & IRR $^{\mathrm{a}}$ & (IC95\%) & $\mathrm{IRR}^{\mathrm{a}}$ & (IC95\%) \\
\hline 1983 a 1992 & $1,336^{b}$ & $(1,296$ a 1,377$)$ & $1,272^{b}$ & $(1,234$ a 1,311$)$ & $1,223^{b}$ & $(1,185$ a 1,277$)$ \\
\hline 1993 a 2002 & 1 & & 1 & & 1 & \\
\hline \multicolumn{7}{|l|}{ Sexo } \\
\hline Masculino & $1,140^{\mathrm{b}}$ & $(1,106$ a 1,176$)$ & $1,304^{b}$ & $(1,265$ a 1,344$)$ & $2,165^{b}$ & $(2,086$ a 2,247$)$ \\
\hline 0 a 14 anos & $0,027^{b}$ & $(0,026$ a 0,029$)$ & $0,140^{b}$ & $(0,133$ a 0,147$)$ & $5 \mathrm{E}-05^{b}$ & (4E-05 a $6 \mathrm{E}-05)$ \\
\hline 15 a 44 anos & $0,030^{\mathrm{b}}$ & $(0,029$ a 0,031$)$ & $0,063^{b}$ & $(0,061$ a 0,066$)$ & $0,016^{b}$ & $(0,015$ a 0,017$)$ \\
\hline 45 a 64 anos & $0,272^{b}$ & $(0,265$ a 0,279$)$ & $0,317^{b}$ & $(0,306$ a 0,328$)$ & $0,261^{b}$ & $(0,251$ a 0,271$)$ \\
\hline 65 a 74 anos & 1 & & 1 & & 1 & \\
\hline \multicolumn{7}{|l|}{ Regiões } \\
\hline Nordeste & 1,026 & $(0,931$ a 1,130$)$ & $1,475^{b}$ & $(1,270$ a 1,715$)$ & $0,695^{b}$ & $(0,613$ a 0,788$)$ \\
\hline
\end{tabular}

${ }^{a} \mid R R=$ razão de taxas de incidência (incidence rate ratio).

${ }^{b}$ Significativo a $0,01(P<0,01)$.

c Variáveis: percentual de pessoas com idade maior ou igual a 25 anos e 12 anos ou mais de estudo, percentual de pessoas com idade maior ou igual a 25 anos e menos de 4 anos de estudo e renda per capita.

dVariáveis: índice de Gini e razão entre a renda dos $10 \%$ mais ricos e a dos $40 \%$ mais pobres.

e Significativo a $0,05(P<0,05)$.

por diagnóstico e tratamento precoce, o maior risco no primeiro período é indicativo de que, na prática, a reorganização do sistema de saúde, procurando priorizar a atenção básica, pode ter repercutido sobre a incidência de doenças diagnosticadas e tratadas precocemente e, em última instância, sobre a mortalidade por essas causas, conforme sugerido por outros estudos $(3,36)$.

Cabe ressaltar que o processo de reorganização do sistema de saúde no Brasil não se iniciou em 1988 com a criação do SUS. O SUS é, na realidade, o resultado de um longo processo político para implementação de propostas de conformação de um modelo público de atenção à saúde. Em virtude da natureza, complexidade e abrangência das mudanças institucionais, políticas e operacionais em desenvolvimento, o SUS está ainda sujeito a aprimoramentos. Todavia, em que pese toda essa complexidade, as mudanças foram mais evidenciadas em diferentes instâncias de estrutu- ração da atenção à saúde no início da década de 1990 (24, 25, 37). Assim, é possível, pelo menos em parte, creditar a essas mudanças o processo de descenso da mortalidade por causas evitáveis por atenção à saúde.

A análise dos dados aqui apresentados demonstrou ainda que, no Brasil, ser do sexo masculino representou um risco maior para qualquer um dos grupos de causas evitáveis analisados, sendo que, no caso da doença isquêmica do coração, esse diferencial al- 
cançou 116,51\%. Em relação à idade, observa-se um risco menor para as populações mais jovens para todos os grupos de causas evitáveis por atenção à saúde, conforme esperado. A localização geográfica também foi significativa, com menor risco para a Região Sul, uma das mais desenvolvidas do País, para as causas evitáveis por diagnóstico e tratamento e para as causas evitáveis por melhoria no tratamento e atenção médica. O Centro-Oeste, onde os riscos foram menores do que na Região Sul (considerada como região de referência), foi exceção, entretanto. Uma explicação pode ser o fato de que um dos municípios dessa região (Brasília) apresentava melhores indicadores sociais e de saúde, os quais se distinguiam da média regional. A situação socioeconômica, expressa nos dois indicadores construídos através de análise fatorial, mostrou uma menor influência no comportamento da mortalidade evitável, possivelmente porque a própria seleção dos municípios, ao se basear em critério de qualidade da informação, selecionou municípios com condições socioeconômicas mais similares.

A partir dessas observações, é possível inferir que a formulação de tipologias de causas de morte evitáveis pode contribuir para a identificação de fatores que afetam a mortalidade. Para a realidade brasileira, em que há ainda marcantes diferenciais regionais e sociais, expressos também nos níveis de mortalidade, esse tipo de abordagem permite analisar a mortalidade por causas evitáveis como indicador da qualidade da assistência de saúde. Apesar de sua relevância, ainda são poucos os estudos desenvolvidos no Brasil que utilizam esse indicador, mais explorado em estudos da mortalidade infantil e perinatal (16-18). Mesmo em outros países da América Latina, também são poucos os estudos nessa linha; a abordagem da mortalidade evitável tem sido mais direcionada para outras classificações, que estabelecem uma categorização das causas de morte segundo o grau de evitabilidade, considerando também causas passíveis de serem evitadas através de ações de saúde pública e melhorias nas condições de vida da população (38-40).
Nos países desenvolvidos, as análises de mortalidade publicadas indicam um padrão consistente de declínio na mortalidade evitável, que se caracteriza por uma velocidade de queda mais acentuada do que a da mortalidade por outras causas $(3,8,41)$. Devido a essas mudanças, existe uma tendência de forte redução da mortalidade proporcional por causas evitáveis. Os estudos sugerem que, pelo menos em parte, o declínio da mortalidade por causas evitáveis se deve à melhoria na atenção à saúde (4, 12-14, 42-44). A qualidade da atenção está relacionada a diferentes elementos, relativos à estrutura organizacional do sistema de saúde, à oferta e ao acesso aos serviços de saúde e aos resultados em termos do impacto na condição individual e coletiva de saúde (36). A relação entre esses diferentes componentes representa um importante recurso para avançar nas avaliações da qualidade da assistência para mais além das análises de tendências e variações da mortalidade evitável. Entretanto, a análise da mortalidade por causas evitáveis pode ser útil para o planejamento de saúde, considerando que oferece um indicador sensível para acompanhar os efeitos de políticas de saúde que preconizem a ampliação e o aprimoramento da atenção à saúde oferecida à população (45). Quanto a esse aspecto, tornase imperioso que sistemas nacionais e locais sejam capazes de identificar quais são as ações que devem ser priorizadas, no sentido de aumentar a capacidade resolutiva das intervenções de saúde para a redução da mortalidade evitável.

É importante lembrar que as diferenças de mortalidade podem, pelo menos parcialmente, ser explicadas pela variação na incidência. Se a incidência pode ser alterada por intervenção médica, variações na incidência indicam possíveis variações na qualidade do cuidado médico (5). Porém, se por um lado as diferenças de tendência na incidência de causas evitáveis devem-se a diferenças na efetividade de certas intervenções (prevenção e tratamento), podem também ser espontâneas e devidas a outros fatores que não a atenção à saúde, como por exemplo a rápida difusão de estilos de vida alternativos e mais saudáveis (14).
Uma conseqüência provável dessas questões é a necessidade de tornar mais complexa a capacidade para avaliar sistemas de saúde, valendo-se de dados agregados de mortalidade. Essa exigência decorre da ampliação do escopo da mortalidade evitável e da redução da extensão de variação à medida que se observa uma tendência de declínio nas taxas de mortalidade por causas evitáveis. Nesse caso, os estudos ecológicos são indicados, pois permitem analisar o impacto de variáveis de contexto, que não têm reprodutibilidade no nível individual. Da perspectiva da saúde pública, a abordagem ecológica pode ser de grande interesse, considerando-se a necessidade de avaliar os efeitos das políticas de saúde (46).

Por outro lado, deve-se considerar ainda que os estudos de mortalidade por causas evitáveis podem apresentar diferenças nos padrões e tendências em razão da própria seleção das causas estudadas. Uma tipologia de causas evitáveis é sempre determinada pelos propósitos da análise. Deve ficar claro que essa escolha vai influenciar os resultados encontrados (9). Portanto, no estudo realizado, é importante considerar que, na seleção de causas de morte, foram identificados aqueles grupos de causas que podem ser considerados como mais suscetíveis a diferentes tipos de atenção médica, tendo em vista o interesse em analisar o impacto das mudanças introduzidas na organização e oferta de serviços de saúde no Brasil. Deve-se ter claro que essa seleção não esgota todas as causas de morte que poderiam também ser consideradas como evitáveis, bem como contempla causas que sofrem a influência de outras intervenções, não restritas ao setor saúde.

Uma limitação para análises de mortalidade por causas no Brasil, já evidenciada em outros estudos (29), refere-se ao sub-registro de óbitos, que ainda persiste como um problema importante em um grande número de municípios. Na seleção dos municípios deste estudo, os indicadores de qualidade da informação (menos de $10 \%$ de causas mal definidas, por exemplo) foram aplicados desde o período do estudo (1983 a 1985), quando os sistemas de informação provavelmente apresen- 
tavam maior grau de sub-registro. Em anos recentes, muitos esforços têm sido desenvolvidos no sentido de produzir estatísticas oficiais de boa qualidade, reduzindo o sub-registro de óbitos e aprimorando a classificação de causas básicas nas declarações de óbitos (47). Esse é um aspecto a ser considerado, pois informações de boa qualidade minimizam os vieses que podem ser introduzidos na análise pela variabilidade de códigos de causas, devido a mudanças nas classificações utilizadas (8), e também por problemas no registro de causas para populações mais velhas, sujeitas a apresentar, em maior grau, causas múltiplas de morte (26).

A partir dos critérios adotados, foram selecionados somente 117 municípios brasileiros. Desses, a grande maioria está localizada no Sudeste e Sul. Por um lado, esse é um desafio para as análises de mortalidade, uma vez que o universo de estudo, muitas vezes, não permite captar as especificidades regionais existentes e por si só já remete a problemas na atenção à saúde. Mas, por outro lado, os municípios selecionados detêm um terço da população brasiChild CG 3rd, Fishman AP, Perrin EB. Measuring the quality of medical care. A clinical method. N Engl J Med. 1976;294(11):582-8.

2. Charlton JR, Hartley RM, Silver R, Holland WW. Geographical variation in mortality from conditions amenable to medical intervention in England and Wales. Lancet. 1983; 1(8326 Pt 1):691-6.

3. Mackenbach JP, Bouvier-Colle MH, Jougla E. "Avoidable" mortality and health services: a review of aggregate data studies. J Epidemiol Community Health. 1990;44(2):106-11.

4. Albert X, Bayo A, Alfonso JL, Cortina P, Corella D. The effectiveness of health systems in influencing avoidable mortality: a study in Valencia, Spain, 1975-90. J Epidemiol Community Health. 1996;50(3):320-5.

5. Treurniet HF, Looman CW, van der Maas PJ, Mackenbach JP. Variations in "avoidable" mortality: a reflection of variations in incidence? Int J Epidemiol. 1999;28(2):225-32.

6. Manuel DG, Mao Y. Avoidable mortality in the United States and Canada, 1980-1996. Am J Public Health. 2002;92(9):1481-4.

7. Andreev EM, Nolte E, Shkolnivkov VM, Varavikova E, McKee M. The evolving pattern of avoidable mortality in Russia. Int J Epidemiol. 2003;32(3):437-46. leira-cerca de 33\% do total, segundo o censo demográfico de 2000. Ao mesmo tempo, são aqueles onde a reorganização do sistema de saúde mais avançou, pois cerca de $70 \%$ deles já se encontravam, em junho de 2005, em um estágio no qual a gestão dos serviços de saúde era de responsabilidade do município, que recebia o total de recursos federais programados para o custeio da assistência em seu território (48).

Assim, pressupondo que, conforme argumenta Chackiel (49), nenhuma informação sobre mortalidade é totalmente desprezível, desde que se conheçam as suas limitações e se saiba o que se pode esperar dela, a análise da mortalidade por causas evitáveis pode ser relevante na discussão sobre a aplicabilidade desse indicador para avaliar a qualidade da atenção médica prestada. Os resultados deste estudo sugerem que o declínio da mortalidade por causas evitáveis no Brasil entre 1983 e 2002 pode ser creditado, em parte, às mudanças na oferta e no acesso a serviços de saúde. Entretanto, o peso relativamente expressivo das causas evitáveis no total de óbitos e os

\section{REFERÊNCIAS}

8. Nolte E, McKee M. Does health care save lives? Avoidable mortality revisited. London: Nuffield Trust; 2004.

9. French KM, Jones K. Impact of definition on the study of avoidable mortality: geographical trends in British deaths 1981-1998 using Charlton and Holland's definitions. Soc Sci Med. 2006;62(6):1443-56.

10. Westerling R, Smedby B. The European Community "avoidable death indicators" in Sweden 1974-1985. Int J Epidemiol. 1992;21(3):502-10.

11. Humblet PC, Lagasse R, Levêque A. Trends in Belgian premature avoidable deaths over a 20 year period. J Epidemiol Community Health. 2000;54(9):687-91.

12. Mackenbach JP, Looman CW, Kunst AE, Habbema JD, van der Maas PJ. Post-1950 mortality trends and medical care: gains in life expectancy due to declines in mortality from conditions amenable to medical interventions in The Netherlands. Soc Sci Med. 1988;27(9):889-94.

13. Charlton JR, Velez R. Some international comparisons of mortality amenable to medical intervention. Br Med J (Clin Res Ed). 1986; 292(6516):295-301.

14. Mackenbach JP, Looman CW, Kunst AE, Habbema JD, van der Maas PJ. Regional differences in decline of mortality from selected conditions: The Netherlands, 1969-1984. Int J Epidemiol. 1988;17(4):821-9. níveis das taxas de mortalidade por essas causas no Brasil podem ser um indicativo de que o sistema de saúde ainda não é suficientemente capaz de enfrentar essa questão, em consonância com os resultados do estudo realizado na Inglaterra nos anos 1970 (2).

Cabe ressaltar ainda a persistência de aspectos não esclarecidos, relacionados possivelmente com diferenciais regionais e socioeconômicos na mortalidade evitável. A questão da eqüidade no acesso aos serviços de saúde deve ser então abordada, tendo em vista que pode ser um dos fatores explicativos para as variações existentes na mortalidade por causas evitáveis entre as regiões e os grupos sociais. Estudos nessa direção podem revelar novos parâmetros para avaliar a importância da atenção à saúde no declínio da mortalidade evitável no Brasil.

Agradecimento. O presente estudo foi parcialmente financiado pela Fundação de Amparo a Pesquisa do Estado de Minas Gerais (processo ${ }^{\circ}$ CDS 1624/98).
15. Pampalon R. Avoidable mortality in Québec and its regions. Soc Sci Med. 1993;37(6): 823-31.

16. Ortiz LP. A mortalidade por causas evitáveis no estado de São Paulo - 1975/1976. Informe demográfico. São Paulo: Fundação SEADE 1980:4:49-109.

17. Silva MGC. Anos potenciais de vida perdidos por causas evitáveis, segundo sexo, em Fortaleza, em 1996-1998. Epidemiol Serv Saude. 2003;12(2):97-108.

18. Caldeira AP, França E, Perpétuo IHO, Goulart EMA. Evolução da mortalidade infantil por causas evitáveis na região de Belo Horizonte de 1984 a 1998. Rev Saude Publica. 2005;39(1): 67-74.

19. Hartz ZMA, Champagne F, Contrandiopoulos A, Leal MC. Avaliação do programa materno-infantil: análise de implantação em sistemas locais de saúde no nordeste do Brasil. Em: Hartz ZMA, org. Avaliação em Saúde dos modelos conceituais à prática na análise da implantação de programas. Rio de Janeiro: Fiocruz; 1997. Pp. 89-131.

20. Lansky S, França E, Leal MC. Mortalidade perinatal e evitabilidade: revisão da literatura. Rev Saude Publica. 2002;36(6):759-72.

21. Barreto ML, Carmo EH. Situação de saúde da população brasileira: tendências históricas, determinantes e implicações para as po- 
líticas de saúde. Inf Epidemiol SUS. 1994; (3/4):5-34.

22. Duchiade MP. População brasileira: um retrato em movimento. Em: Minayo MCS, org. Os muitos Brasis: saúde e população na década de 80. São Paulo/Rio de Janeiro: Hucitec/ABRASCO; 1995. Pp. 14-56.

23. Almeida CM. Reforma del Estado y reforma de sistemas de salud. Cuadernos Medico Sociales. 2001;79(1):27-58.

24. Brasil, Ministério da Saúde. Sistema Único de Saúde (SUS): princípios e conquistas. Brasília: Ministério da Saúde; 2001.

25. Brasil, Ministério da Saúde. Normas operacionais básicas do Sistema Único de Saúde: NOB-SUS 01/91, NOB-SUS 01/93, NOB-SUS 01/96. Brasília: Ministério da Saúde;1997.

26. Santo AH. Equivalência entre revisões da Classificação Internacional de Doenças: causas de morte. Rev Saude Publica. 2000;34(1):21-8.

27. Haraki CAC, Gotlieb SLD, Laurenti R. Confiabilidade do Sistema de Informações sobre Mortalidade em município do sul do Estado de São Paulo. Rev Bras Epidemiol. 2005;8(1):19-24.

28. Szwarcwald CL, Leal MC, Andrade CLT, Souza Jr. PRB. Estimação da mortalidade infantil no Brasil: o que dizem as informações sobre óbitos e nascimentos do Ministério da Saúde? Cad Saude Publica. 2002;18(6):1725-36.

29. Santo AH. Avaliação da qualidade de codificação das causas de morte no Estado de São Paulo, Brasil. Inf Epidemiol SUS. 2000;9(3): 189-98.

30. Simonato L, Ballard T, Bellini P, Winkelmann R. Avoidable mortality in Europe 1955-1994: a plea for prevention. J Epidemiol Community Health. 1998;52(10):624-30.

31. Tobias M, Jackson G. Avoidable mortality in New Zealand, 1981-97. Aust N Z J Public Health. 2001;25(1):12-20.
32. Wigglesworth JS. Classification of perinatal deaths. Soz Praventivmed. 1994;39(1):11-4.

33. Ortiz LP. A utilização das causas evitáveis na mortalidade infantil como instrumento de avaliação das ações de saúde. Em: Anais do X Encontro Nacional de Estudos Populacionais, 1996, Out 7-11; Caxambu, MG. Belo Horizonte: ABEP, 1996. Pp. 2253-68.

34. Programa das Nações Unidas para o Desenvolvimento (PNUD), Instituto de Pesquisa Econômica Aplicada (IPEA), Fundação João Pinheiro. Atlas do Desenvolvimento Humano no Brasil. 2000. http://www.pnud.org.br/ atlas/. Acessado em 20 de julho de 2005.

35. Long JS, Freese J. Models for count outcomes. Em: Long JS, Freese J. Regression models for categorical dependent variables using Stata. Texas: Stata; 2001. Pp. 223-62.

36. Bixby LR. Evaluación del impacto de la reforma del sector de la salud en Costa Rica mediante un estudio casi experimental. Rev Panam Salud Publica. 2004;15(2):94-103.

37. Levcovitz E, Lima LD, Machado CV. Política de saúde nos anos 90: relações intergovernamentais e papel das Normas Operacionais Básicas. Cienc Saude Coletiva. 2001; 6(2):269-91.

38. Taucher E. La mortalidad en Chile desde 1955 a 1975; tendencias y causas. Notas de Pobl. 1978;6(18):113-42.

39. Gattini C, Sanderson C, Castillo-Salgado C. Variación de los indicadores de mortalidad evitable entre comunas chilenas como aproximación a las desigualdades de salud. Rev Panam Salud Publica. 2002;12(6):454-61.

40. Noriega Bravo VM, Astraín Rodríguez ME. Mortalidad evitable según condiciones de vida en adultos del municipio la Lisa, 19961998. Rev Cubana Salud Publica. 2004;30(4): $319-25$.
41. Poikolainen K, Eskola J. The effect of health services on mortality: decline in death rates from amenable and non-amenable causes in Finland, 1969-81. Lancet. 1986;1(8474):199-202.

42. Niti M, Ng TP. Temporal trends and ethnic variations in amenable mortality in Singapore 1965-1994: the impact of health care in transition. Int J Epidemiol. 2001;30(5):966-73.

43. Nolte E, Scholz R, Shkolnikov V, McKee M. The contribution of medical care to changing life expectancy in Germany and Poland. Soc Sci Med. 2002;55(11):1905-21.

44. Nolte E, McKee M. Measuring the health of nations: analysis of mortality amenable to health care. BMJ. 2003;327(7424):1129.

45. Lourenço PMC. Impacto da assistência médica sobre a mortalidade: um estudo da variação geográfica da mortalidade evitável nas capitais brasileiras (1979-1992) [tese]. São Paulo: Universidade de São Paulo; 1997.

46. Diez Roux AV. The study of group-level factors in epidemiology: rethinking variables, study designs and analytical approaches. Epidemiol Rev. 2004;26:104-11.

47. Vasconcelos AM. A qualidade das estatísticas de óbitos no Brasil. Rev Bras Estudos Pop. 1998;15(1):115-24.

48. Brasil, Ministério da Saúde, Departamento de Apoio à Descentralização, Comissão Intergestores Tripartite. Relação nominal de municípios habilitados por Estado, jun/2005. http:// dtr2001.saude.gov.br/dad/index.htm. Acessado em 29 de agosto de 2005.

49. Chackiel J. La investigación sobre causas de muerte en la América Latina. Notas Poblacion. 1987;44:9-30.

Manuscrito recebido em 1 de março de 2006. Aceito em versão revisada em 18 de abril de 2007.
ABSTRACT

The relationship between deaths that are avoidable with adequate health care and the implementation of the Unified Health System in Brazil

\footnotetext{
Key words Health care reform, health services research, mortality, cause of death, risk factors,
}

Brazil. avoidable with adequate health care and the reorganization of the Brazilian health care system between 1983 and 2002.

Method. This ecological study analyzed avoidable mortality in 117 municipalities of Brazil. The causes of death avoidable with adequate health care were grouped into three: (1) ones avoidable through early diagnosis and treatment, (2) ones avoidable with improvements in the quality of treatment and medical care, and (3) ischemic heart disease. To evaluate the association between avoidable mortality and reorganization of the health care system, the period under study was divided into two subperiods: from 1983 through 1992 and from 1993 through 2002 (respectively, before and after approval of the operational guideline that served as the reference for the organization of the Unified Health System (Sistema Único de Saúde)). A negative binomial regression model that controlled for sex, age, geographic region, and socioeconomic conditions was used for the analysis. Results. During the period analyzed, 1854165 individuals between 0 and 74 years old died from avoidable causes in the municipalities studied. The multivariate analysis showed that, for all three groups of avoidable causes, the risk of avoidable mortality was higher in the 1983-1992 subperiod than in the 1993-2002 subperiod. For the entire 1983-2002 period, the risk was higher for males than for females, especially with respect to ischemic heart disease. Younger populations had lower risk. Higher socioeconomic level reduced the risk of death from avoidable causes, except for ischemic heart disease. Conclusions. Our results suggest that in Brazil the decrease in avoidable mortality from the 1983-1992 subperiod to the 1993-2002 subperiod was partially due to the changes in the availability of and access to health services brought about by the reorganization of the Brazilian health care system. 